\title{
Primary kidney parenchyma squamous cell carcinoma mimicking xanthogranulomatous pyelonephritis: A case report
}

\author{
ZHAO WANG ${ }^{1,2}$, BIN YAN $^{1}$, YONG-BAO WEI ${ }^{1,3}$, NA HU $^{4}$, QIN SHEN $^{5}$, \\ DUO LI ${ }^{6}, \mathrm{JIN}^{-R U I} \mathrm{YANG}^{1 *}$ and XIN YANG ${ }^{7^{*}}$ \\ ${ }^{1}$ Department of Urology, The Second Xiangya Hospital, Central South University, Changsha, Hunan 410011; \\ ${ }^{2}$ Department of Urology, Xiangya Hospital, Central South University, Changsha, Hunan 410008; ${ }^{3}$ Department of Urology, \\ Fujian Provincial Hospital, The Teaching Hospital of Fujian Medical University, Fuzhou, Fujian 350001; \\ Departments of ${ }^{4} \mathrm{PET} / \mathrm{CT}$ Center, ${ }^{5}$ Radiology, ${ }^{6}$ Pathology and ${ }^{7}$ Oncology, \\ The Second Xiangya Hospital, Central South University, Changsha, Hunan 410011, P.R. China
}

Received December 17, 2014; Accepted January 22, 2016

DOI: $10.3892 / \mathrm{ol} .2016 .4200$

\begin{abstract}
Primary kidney parenchyma squamous cell carcinoma is extremely rare, and this is the forth case to be reported. In the present study, a case of a 61-year old man is discussed. The man presented with recurrent lumbago, gross hematuria for nearly 2 months, and suspicious inflammatory kidney diseases on contrast-enhanced computed tomography (CT) and fludeoxyglucose-positron emission tomography (FDG-PET)/CT, but a tumor can not be excluded completely prior to surgery. Finally, radical nephrectomy was performed, and histological analysis determined that the diagnosis was kidney parenchyma squamous cell carcinoma with inflammation invasion. The present case highlights the potential confusion of preoperative diagnosis of renal tumor with inflammation, and introduces the potential role of FDG-PET in its diagnosis and survival evaluation in renal malignancies.
\end{abstract}

\section{Introduction}

Kidney squamous cell carcinomas are rare occurrences in renal malignancies, particularly for kidney parenchyma squamous cell carcinoma (1-5). To the best of our knowledge, only three cases of patients with kidney parenchyma cell carcinoma have been reported since December 2014 (2-5).

Correspondence to: Professor Jin-Rui Yang, Department of Urology, The Second Xiangya Hospital, Central South University, 139 Ren Min Road, Changsha, Hunan 410011, P.R. China

E-mail: yjinrui@yahoo.com

Mr. Xin Yang, Department of Oncology, The Second Xiangya Hospital, Central South University, 139 Ren Min Road, Changsha, Hunan 410011, P.R. China

E-mail: xinyangoncolo@163.com

*Contributed equally

Key words: kidney, squamous cell carcinoma, FDG-PET, malignancy, prognosis
The three cases consisted of a 73-year old man (2), a 51-year old man (5) and a 60-year old woman (4). All the patients underwent nephrectomies. The 73-year old man was followed up 3 months after surgery, the 51-year old man was followed up 6 and 12 months after surgery and the 60-year old woman was followed up 13 months after surgery; all patients remained alive with no evidence of disease at the time of follow-up (2-5). The present study reports a case of kidney parenchyma squamous cell carcinoma with inflammation invasion, which mimicked xanthogranulomatous pyelonephritis on radiological examination; the literature concerning kidney squamous cell carcinomas is also reviewed.

\section{Case report}

A 61-year old man presented to the Second Xiangya Hospital (Changsha, China) in October 2014 with right lumbago and gross hematuria that had been present for nearly 2 months. The patient had right renal parenchyma lithotomy $\sim 14$ years ago. Physical examination demonstrated positive percussion pain on the right kidney region. Palpation on the kidneys did not reveal any pathological symptoms.

On admission, routine urine tests demonstrated that the urine was clearly positive for white blood cells (WBCs) (WBC count, 1,187.50/ $\mu \mathrm{l}$; normal range, $0-25 / \mu \mathrm{l})$. C-Reactive protein measured $72.20 \mathrm{mg} / \mathrm{l}$ (normal, $<8 \mathrm{mg} / \mathrm{l}$ ), erythrocyte sedimentation rate was $81 \mathrm{~mm} / \mathrm{h}$ (normal, $<15 \mathrm{~mm} / \mathrm{h}$ ), procalcitonin was $3.23 \mathrm{ng} / \mathrm{ml}$ (normal, $<0.05 \mathrm{ng} / \mathrm{ml}$ ). Renal and liver function tests results were within the normal ranges. Urine cytology examination demonstrated massive epithelial cells and neutrophils without malignancies. Contrast-enhanced computed tomography (CT) was performed using a SOMATOM Sensation CT scanner (Siemens, Munich, Germany). It demonstrated enlargement of the right kidney with abnormal morphology and dilatation of the ureter, multiloculated cyst-like masses with soft tissues, and perirenal fatty space was fuzzy (Fig. 1A and B). The lesions observed on the CT scan were atypical and were considered as xanthogranulomatous pyelonephritis or tuberculosis; however, the presence of a rare renal tumor could not be completely excluded. Tuberculosis tests 

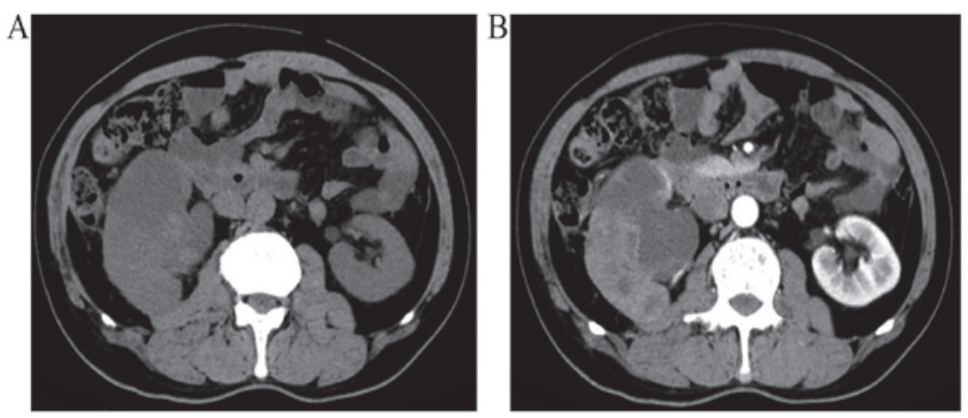

Figure 1. CT images of the patient. (A) Normal and (B) enhanced CT scans demonstrated the enlargement of the right kidney with abnormal morphology, multiple cyst-like masses with soft tissues and the perirenal fatty space was observed to be fuzzy. CT, computed tomography.

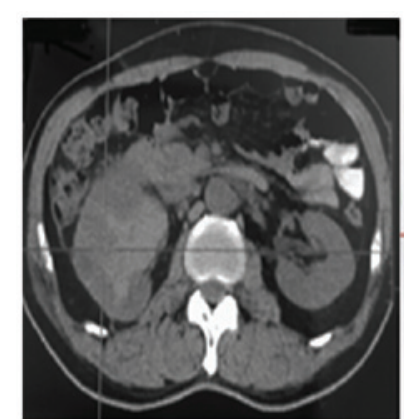

CT

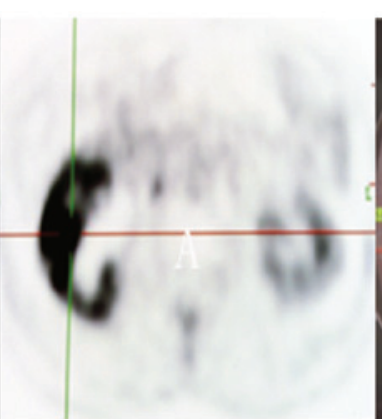

PET

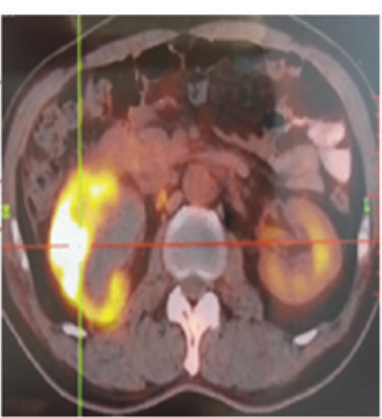

FUSION

Figure 2. FDG-PET images. The images demonstrate hydronephrosis with fuzzy perirenal fatty space, and FDG was taken up by cystic-solid mixed masses in the right kidney. CT, computed tomography; PET, positron emission tomography; FDG, fludeoxyglucose.
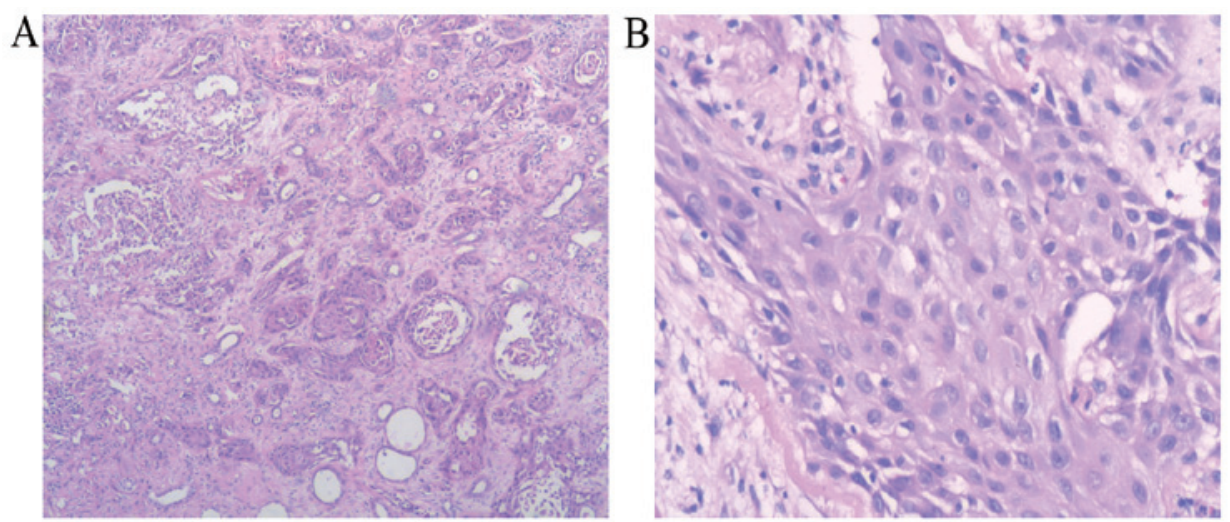

Figure 3. Histological examination. Hematoxlyin and eosin staining at (A) x40 and (B) x200 magnification. A massive invasion of inflammatory cells was observed, which is a hallmark of squamous cell carcinoma of the renal parenchyma.

[purified protein derivative (PPD)-immunoglobulin (Ig)M, PPD-IgG and MycoDot] were performed and were negative.

In total, 10 days following admission, the patient had a fever (highest temperature, $39.6^{\circ} \mathrm{C}$ ) and chills, and blood rountine tests demonstrated a white blood cell count of $16.94 \times 10^{9}$ cells/ 1 (normal range, $3.50-9.50 \times 10^{9}$ cells/1) and a neutrophil percentage of $92.34 \%$ (normal range, 40.00-75.00\%). Therefore, physicians hypothesized that the patient had septicemia. Antibiotics were prescribed (latamoxef for 2 week, $1 \mathrm{~g}$ twice a day, intravenous combined with moxifloxacin for 5 days, $0.4 \mathrm{~g}$ once a day, intravenous), which were adjusted (imipenem cilastatin sodium, 0.5 g every $6 \mathrm{~h}$, intravenous) according to a routine blood test (procalcitonin and C-reactive protein) and a routine urine examination, including color, glucose and protein concentration, $\mathrm{pH}$ and white and red blood cell count, urine culture and advice from clinical pharmacists. After septicemia was controlled in the patient, cystoscopy was performed. Fludeoxyglucose-positron emission tomography (FDG-PET; Biography mCTx; Siemens) was also performed following the administration of $9.43 \mathrm{mCi}$ FDG. Cystoscopy did not reveal the presence of carcinoma in the bladder and urethra. FDG-PET showed hydronephrosis with a fuzzy perirenal fatty space, and valid FDG was taken up by cystic-solid mixed masses in the right kidney (Fig. 2). The lesions were considered to be renal malignancies, but renal inflammation diseases could not be excluded completely. 
Right radical nephrectomy was performed. Histological examination using hematoxylin and eosin staining (Sinopharm Chemical Reagent Co., Ltd., Shanghai China) on paraffin-embedded tissues under a microscope (BX51TF; Olympus Corporation, Tokyo, Japan) demonstrated moderate-differentiated squamous cell carcinoma of the renal parenchyma with a massive invasion of inflammatory cells (Fig. 3A and B). The patient was discharged 10 days following surgery. Follow-up one month after surgery in December 2014 showed that the patient had no febrile, gross hematuria or abnormal abdominal signs. Written informed consent was obtained from the patient for the publication of the present study.

\section{Discussion}

Kidney squamous cell carcinomas are rare renal malignancies, and are classified as renal parenchyma and pelvic squamous cell carcinomas according to where they arise. Primary kidney pelvic squamous cell carcinomas account for $0.5-0.8 \%$ in kidney malignancies (1). Renal parenchyma squamous cell carcinomas are extremely rare; to the best of our knowledge only 3 cases have been reported until now (2-5).

Urinary calculi and chronic inflammation are some of the important factors associated with renal squamous cell carcinomas $(4,5)$. In the present case, the patient has a history of kidney calculi for $>14$ years and surgery was performed to remove the stone. The patient exhibited chronic urinary inflammation and antibiotics were intermittently prescribed. These are two predisposing factors associated with the development of squamous cell carcinomas (4-5).

Ultrasound and CT are important tools to evaluate masses in renal malignancies. Xanthogranulomatous pyelonephritis, secondary malignancies should be taken into account in order to achieve a differential diagnosis for renal masses (1). In the present case, contrast-enhanced $\mathrm{CT}$ revealed the presence of lesions in the right kidney. According to the patient's history and primary blood and urine tests, xanthogranulomatous pyelonephritis was one of the most consistent diagnosis. Difficulties exist in evaluating primary renal masses and achieving differential diagnosis in radiological examinations prior to surgery due to the nonspecific features of these lesions (6).

FDG-PET/CT has been verified as an effective tool in diagnosis, preoperative and prognosis evaluation in renal tumors $(7,8)$. A meta-analysis previously demonstrated that the sensitivity and specificity of FDG-PET for renal lesions are 62 and $88 \%$, respectively; however, sensitivity and specificity increase to 84 and $91 \%$ for extra-renal lesions (9). FDG-PET is reportedly more consistent in detecting extra-renal lesions than renal lesions (9). In the present case, FDG-PET/CT was performed prior to surgery. Since the kidney has an abnormal structure and if invasive inflammation has occurred, it is difficult to make a definite diagnosis prior to surgery. Sometimes renal benign diseases resemble renal malignancies, such as acute pyelonephritis, xanthogranulomatous pyelonephritis or inflammatory pseudotumors on FDG-PET/CT (10-12).
Maximum standardized uptake value $\left(\mathrm{SUV}_{\max }\right)$ in FDG-PET/CT is a potential measurement that may be used to evaluate patients' survival in renal cell carcinoma (8). For the patient in the present case, the $\mathrm{SUV}_{\max }$ of the early image was 19.9, and 33.8 in the delayed image. According to Ferda et al (8) 12 month-mortality rate may be as high as $62.5 \%$. Follow-up for the patient is therefore extremely important.

Primary kidney parenchyma squamous cell carcinoma is rare. The present study introduces a case of primary kidney parenchyma squamous cell carcinoma in a 61-year old man, which was initially diagnosed as xanthogranulomatous pyelonephritis on CT. In addition, FDG-PET/CT did not distinguish renal inflammation diseases from various types of tumors in the present case. Right radical nephrectomy was performed, and histological diagnosis determined a case of kidney parenchyma squamous cell carcinoma with inflammation invasion. The 1 year mortality for this case may reach as high as $62.5 \%$ according to the $\mathrm{SUV}_{\max }$. In conclusion, FDG-PET/CT is critical in assisting with the diagnosis and prognosis evaluation in kidney malignancies.

\section{References}

1. Kalayci OT, Bozdag Z, Sonmezgoz F and Sahin N: Squamous cell carcinoma of the renal pelvis associated with kidney stones: Radiologic imaging features with gross and histopathological correlation. J Clin Imaging Sci 3: 14, 2013.

2. Terada T: Synchronous squamous cell carcinoma of the kidney, squamous cell carcinoma of the ureter and sarcomatoid carcinoma of the urinary bladder: A case report. Pathol Res Pract 206: 379-383, 2010.

3. Pusiol T, Zorzi MG and Morini A: Comment on: Primary squamous cell carcinoma of the renal parenchyma. Indian J Pathol Microbiol 56: 70, 2013.

4. Kulshreshtha P, Kannan N, Bhardwaj R and Batra S: Primary squamous cell carcinoma of the renal parenchyma. Indian J Pathol Microbiol 55: 370-371, 2012.

5. Ghosh P and Saha K: Primary intraparenchymal squamous cell carcinoma of the kidney: A rare and unique entity. Case Rep Pathol 2014: 256813, 2014.

6. Bhaijee F: Squamous cell carcinoma of the renal pelvis. Ann Diagn Pathol 16: 124-127, 2012.

7. Oyama N, Ito H, Takahara N, Miwa Y, Akino H, Kudo T, Okazawa H, Fujibayashi Y, Komatsu K, Tsukahara K and Yokoyama O: Diagnosis of complex renal cystic masses and solid renal lesions using PET imaging: Comparison of 11C-acetate and 18F-FDG PET imaging. Clin Nucl Med 39: e208-e214, 2014.

8. Ferda J, Ferdova E, Hora M, Hes O, Finek J, Topolcan O and Kreuzberg B: 18F-FDG-PET/CT in potentially advanced renal cell carcinoma: A role in treatment decisions and prognosis estimation. Anticancer Res 33: 2665-2672, 2013.

9. Wang HY, Ding HJ, Chen JH, Chao CH, Lu YY, Lin WY and Kao CH: Meta-analysis of the diagnostic performance of [18F]FDG-PET and PET/CT in renal cell carcinoma. Cancer Imaging 12: 464-474, 2012.

10. McCammack KC, Hawkes NC, Silverman ED and Paz DA: PET/CT appearance of acute pyelonephritis. Clin Nucl Med 38: e299-e301, 2013.

11. Cheng G, Torigian DA and Alavi A: FDG PET/CT and MRI findings in a patient with focal xanthogranulomatous pyelonephritis mimicking cystic renal malignancy. Clin Nephrol 76: 484-486, 2011.

12. Lee JH, Lee KG, Park HK, Song SY, Kim JY, Kim YH, Choi YY, Jang KS and Park MH: Inflammatory pseudotumor of the kidney mimicking malignancy on 18F-FDG PET/CT in a patient with diabetes and hepatocellular carcinoma. Clin Nucl Med 37: 699-701, 2012. 